\title{
Uncertainty management during conceptual modelling: a cognitive mapping case study
}

\author{
$\underline{\text { L.A. Macadam }}^{\mathrm{a}}$ \\ ${ }^{a}$ Integrated Catchment Assessment and Management (iCAM) Centre, Fenner School of Environment and \\ Society, the Australian National University, ACT \\ Email: leila.macadam@anu.edu.au
}

\begin{abstract}
Scoping' and 'problem framing' begin the integrated assessment and modelling (IAM) process and result in a conceptual model that frames the system of interest according to a defined problem. A conceptual model is mainly qualitative and provides a basis for the later development of a numerical model. Given the significant complexities of interaction among the cross-disciplinary system components that IAM explores, this process of abstracting reality to a more manageable model requires subjective assumptions and decisions tailored to the specific modelling question and purpose. This abstraction and subjectivity may cause a loss of confidence by policy-makers planning to base decisions on model results. To avoid this, best practice guidance from the literature focuses on process and makes recommendations to engage stakeholders, avoid ambiguity in problem framing, maintain transparency on model decisions, and avoid overly-complex model representations that waste resources. The focus on stakeholder engagement and transparency reflects a belief that, ultimately, model utility is largely defined by the acceptance of its outputs by stakeholders, which will be facilitated by an understanding of the modelling process. What is often not clear is at what point each of these recommendations has been addressed sufficiently for the modelling process to progress. In addition, non-modellers may find it difficult to understand the implications of ambiguous problem framing and a lack of model parsimony. This reduces transparency as well as their ability to contribute on multi-disciplinary, multi-sectoral modelling projects.
\end{abstract}

This paper suggests that the concept of uncertainty may be a central driver of best practice in the early phases of scoping, problem framing and conceptual modelling. To illustrate, an existing uncertainty management framework (UMF) is used to guide decisions leading towards a conceptual model for a Cambodian groundwater use case study. Two iterations of the UMF application are performed, alternately using the 'scoping' and 'problem framing' phases as sources of uncertainty to be managed. The first iteration triggered the decision to undertake formal stakeholder engagement to collect additional knowledge about the system to be modelled, and the selection of Eden's cognitive mapping approach to structure and analyse this data. This new information helped to reduce or ignore the iteration 1 uncertainties, as well as facilitate the identification of second-iteration uncertainties (only a selection is illustrated).

Strengths of the UMF approach as applied to the case study included the iterative identification and treatment of uncertainties, its structured, action-oriented, step-by-step nature, and the guidance and flexibility on the choice of methods. Implementation challenges were mainly peripheral, such as choosing how uncertainties should be prioritized, choosing how to prompt the identify task, and sourcing appropriate methods for a given level and nature of uncertainty. Another challenge was communicating the concept of uncertainty to nonmodellers. In terms of handling ambiguity, driving transparency and pursuing parsimony, the uncertainty management approach a) encouraged clarity on what options were available to handle ambiguity and how it might affect problem framing; b) provided transparency through an iterative, structured process that communicated uncertainties about the modelling choices to be made; and c) encouraged questioning of assumptions about model structure, multiple pathways, and which concepts should be made explicit in the conceptual model. The deliberation, transparency, and awareness of resource limits encouraged by the UMF all generated confidence that the resulting system abstraction was defensible and 'enough' to progress through the model-building process. It is hoped that the utility and relative simplicity of this approach as demonstrated with this case study will encourage a stronger and more explicit focus on uncertainty during the scoping, problem framing and conceptualization phases of IAM.

Keywords: Conceptual model, integrated assessment and modelling, cognitive map, uncertainty 


\section{INTRODUCTION}

Integrated assessment and modelling (IAM) is designed to answer questions about complex, multi-issue problems via a modelling approach that intentionally considers integration across the dimensions of space and time, and relevant human and natural settings (Hamilton et al., 2015). Models abstract reality to a manageable representation, a process that requires assumptions and decisions about how phenomena are represented both qualitatively and quantitatively (Jakeman et al., 2006; Robinson, 2015). Each of these assumptions and simplifications involves a level of uncertainty about how it will affect the applicability of the resulting representation and its ability to simulate the reality of interest. Refsgaard (2007, p1555) defines uncertainty as "the degree of confidence that a decision-maker has about possible outcomes and/or probabilities of these". IAM takes an iterative, multi-phase, multi-method approach to this process of abstraction (Hamilton et al., 2015) beginning with 'scoping' and 'problem framing' to inform a mainly qualitative conceptual model that can be used to highlight key processes, explore and test ideas, identify knowledge gaps and check causality . At this stage, system structure is represented by concepts such as entities, processes, stores, funds and stocks, flows, causes and responses linked by explicit and implicit relationships, which may be represented by formalisms ranging from the highly informal to the highly ordered and structured. A good conceptual model informs the 'problem formulation' phase creation of targeted, useful numerical models (Argent et al., 2016).

Jakeman et al. (2006), Hamilton et al. (2015), Argent et al. (2016) and Robinson (2015) provide guidance for best practice IAM and conceptual modelling and emphasize the importance of engaging stakeholders to make sense of the problem by identifying their issues of concern, system boundaries, relevant indicators and management options. This focus on stakeholder involvement is in response to the subjectivity and uncertainty inherent in abstracting a complex system where there is limited data available to describe interactions and arguably no unique and objective problem to be modeled in response to a given question (Brugnach et al., 2008). It also reflects the belief that, ultimately, model utility is largely defined by stakeholder acceptance of its findings. What is little discussed in the best-practice guidance is to what extent stakeholder disagreements on problem framing must be managed (ambiguity), how to set appropriate expectations around the complexity of representation to be incorporated into the model (parsimony), and how much stakeholder communication and consultation is 'enough' at each phase of model development (transparency). This paper suggests these challenges can be addressed by making uncertainty management central to scoping, problem framing and conceptual modelling. In contrast to the more common practice of quantifying uncertainty within model results only, uncertainty can be used as a basis for triggering best practice from the early stages of model building. This is illustrated by applying the uncertainty management framework (UMF) proposed by Guillaume et al. (2012) to a Cambodian case study, a process that triggers stakeholder engagement and cognitive mapping analysis. Previous applications of the UMF have been at latter modelling phases with the assumption that problem framing is complete and in consensus.

\subsection{Handling ambiguity}

Different stakeholders, modellers and experts from different disciplines may bring different concepts and world-views (Argent et al. 2016) to the modelling process, which are often influenced by factors such as values, prior knowledge and disciplinary silos (Brugnach et al. 2008; Hamilton et al. 2015). These biases can result in multiple framings of what problem is being explored, particularly for complex systems. A clearly defined problem definition and model objective is central to IAM (Jakeman et al. 2006) and without acknowledgement and management during scoping and problem framing, this mismatch of expectation about what is to be modelled creates ambiguity, a type of uncertainty arising from the situation where a piece of information can be associated with entirely different meanings (Brugnach et al. 2008). Ignoring this uncertainty is detrimental to transparency and can result in stakeholders and even other members of a multi-disciplinary project team distrusting a model due to the perception that it is answering the 'wrong' question (Eden 1994).

\subsection{Driving transparency}

The difficulty and potential inappropriateness of validating large, integrated models through comparison with real-world data makes it particularly important to build trust in the modelling process with policy makers and others basing decisions on model results (Argent et al. 2016; Jakeman et al. 2006). Transparency builds this trust, avoids the perception of a 'black box' model and ensures that users, stakeholders and other experts who may wish to repurpose knowledge in the future interpreted the model and its results in the right context (Hamilton et al. 2015). In practice, a limitation is that significant communication resources and stakeholder cooperation are required to drive transparency. 


\subsection{Pursuing parsimony}

Best practice models should aim for parsimony by including enough complexity to mimic the processes of interest but no more than is required to meet the model objectives. This is important because more complexity requires more resources to support its implementation, such as supporting data, time and computing power (Jakeman et al., 2006). It can also introduce additional uncertainty in the model results. The pursuit of parsimony begins with clear modelling objectives. These then inform the first-pass assumptions and simplification that occur during conceptual modelling regarding the level of detail and boundaries on what is included and excluded, including on time and spatial scales (Jakeman et al., 2006; Robinson, 2015). If stakeholders do not appreciate the importance of parsimony then there may be resistance to negotiating these necessary assumptions and simplifications, resulting in overly complex models. As Jakeman et al. (2006, p604) state, "better a useful answer to a simple question than too uncertain an answer to a more ambitious question".

\subsection{The uncertainty management framework}

Guillaume (2014) suggests two approaches to managing uncertainty, an uncertainty management framework (UMF) and the Iterative Closed Question Modelling methodology. The UMF is selected for application in this paper since it takes a directed stepby-step approach, encourages documentation and is achievable without additional modelling or engagement resources. The UMF is shown in Figure 1. Firstly the uncertainty should be identified, at which point it should be characterized by its type as defined by the three dimensions of source, nature and level (Walker et al., 2003). These dimensions are then considered when choosing methods to manage a given uncertainty according to the remaining tasks of prioritizing resources to address it (or not), reducing the uncertainty where necessary, describing it, propagating it through the analysis, and communicating it to decision makers. The last task is anticipating actions to manage residual uncertainties (Guillaume et al., 2012).

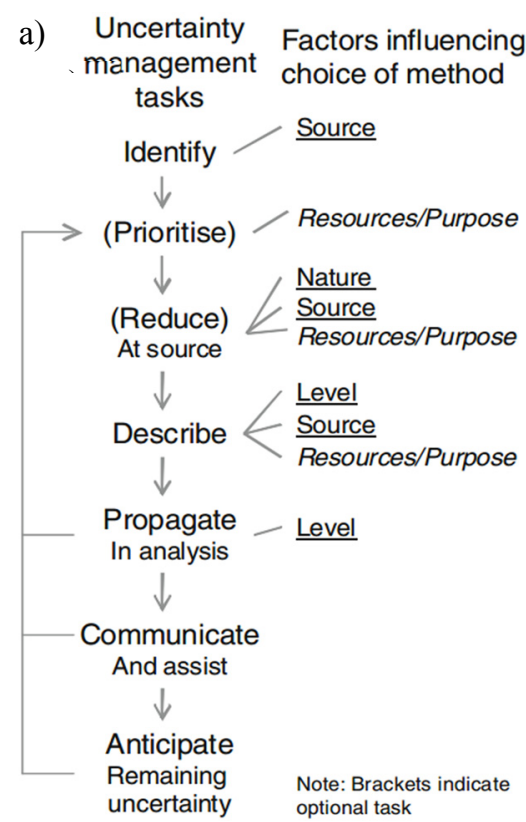
b) Nature: Whether uncertainty can be reduced -Variability also known as irreducible, stochastic or ontological uncertainty -Limited knowledge also known as reducible or epistemic uncertainty - Contradiction also known as ambiguity
Level:
A continuum of detail of knowledge of uncertainty Certainty
Distribution
Bounds
Incl. extreme case scenarios
Scenario
Where not all possible
outcomes are known
Recognised ignorance
Unrecognised unknowns

Source: where the uncertainty comes from

Figure 1. Uncertainty management framework including a) seven uncertainty management tasks, b) uncertainty typology in the three dimensions, which inform choices of methods. A detailed description of the management tasks can be found in Guillaume et al. (2012).

\section{METHODS}

\subsection{Case study}

An expansion of groundwater irrigation in south-east Cambodia has caused concern regarding the sustainability of domestic water supply if groundwater levels drop below the reach of household pumps (Erban and Gorelick, 2016). Rather than waiting for infrastructure to provide surface water access, semi-subsistence farmers are proactively investing in tubewells and pumps in their fields in the hope that increased cropping intensity will generate higher profits. The question is, will groundwater irrigation practices continue to expand, and given the difficulty of exclusion from common pool resources, what might be the positive and negative impacts? These questions require physical, economic and social dimensions to be considered across space and time, prompting an IAM approach. An exploratory model was proposed to simulate future scenarios and inform policy options to address potential tradeoffs.

\subsection{Applying the uncertainty management framework}

Two iterations of the UMF application are performed on the case study, using the 'scoping phase' and 'problem framing phase' as sources of uncertainty to be managed. The first iteration triggered the decision to undertake 
formal stakeholder engagement to collect additional data about the system to be modelled, and the selection of Eden's cognitive mapping approach to structure and analyse this data.

\subsection{Literature review and stakeholder engagement}

An initial literature review was undertaken to identify aspects of the system that were ambiguous or lacking information. Uncertainties arising from this activity informed the stakeholder engagement. Eight national-level resource managers and agricultural development practitioners (referred to as 'resource managers') were then selected by snowball sampling, whereby participants are identified by a referral process that starts with an initial subject. Due to the difficulty of bringing key stakeholders together in a workshop setting, the engagement was achieved by interview. Each resource manager was engaged for a semi-structured interview where they shared knowledge and opinions on the threats and opportunities associated with groundwater use by rural agricultural households in the case study region.

\subsection{Cognitive mapping}

Eden's cognitive mapping approach (Eden, 1994) was selected to analyse the data collected during stakeholder engagement. Each map seeks to represent an individual's perspective on why and how a problem situation has developed. Text analysis identifies action-oriented concepts (which may be bipolar, read as $a$... rather than b), which are mapped as graphical nodes, and the causal relationships among them, represented by positive or negative arrows. Cognitive mapping enables a structural analysis of what concepts are perceived to be central to the problem situation, and then comparison of these perceptions across individual maps. This type of analysis was judged to be a useful method of handling the national-level manager and practitioner engagement data given the expectation of ambiguous stakeholder perspectives and the need to make parsimonious decisions about what key concepts to include in a conceptual model. Following elicitation of knowledge during the interview, Banxia's Decision Explorer software was used to create cognitive maps of content from each individual interview. The content of these maps was then compared and contrasted to generate combined maps demonstrating the most common perspectives found among the interviewees.

\section{RESULTS}

\subsection{First iteration of uncertainty management framework: scoping}

The initial literature review identified some ambiguity of perspective on whether groundwater irrigation was a threat or an opportunity for rural livelihood improvement. Also identified was a lack of detail on the underlying, contextual differences in assumptions about perceived system structure and function that need to be clarified so that transparent and parsimonious choices can be made about how the system is conceptualised. These observations gave rise to identifying three main uncertainties to be handled using the UMF (see Table 1): U11 system behavior; U1-2 processes of interest; and U1-3 future conditions. The first uncertainty is of 'contradiction' nature, since there is an indication of disagreement on whether groundwater poses a risk or opportunity to livelihoods. The nature of the second and third uncertainties is 'limited knowledge' given that more issues of concern and future drivers of change might emerge that could aid in setting boundaries on the

Table 1. Uncertainty management applied to scoping phase

\begin{tabular}{|c|c|c|}
\hline & Uncertainty Type & Action for tasks \\
\hline U1-1 & $\begin{array}{l}\text { Source: representation of system behaviour } \\
\text { Uncertainty: what perceptions suggest } \\
\text { groundwater is a livelihood risk vs an } \\
\text { opportunity? } \\
\text { Nature: contradiction } \\
\text { Level: scenario }\end{array}$ & $\begin{array}{l}\text { Prioritize: central to model linkages, so seek more information } \\
\text { Reduce: by stakeholder engagement on the topic of agricultural groundwater use } \\
\text { Describe: identification of multiple possible linkages between concepts } \\
\text { Propagate: not yet defined } \\
\text { Communicate: acknowledge as current limitation } \\
\text { Anticipate: plan stakeholder engagement with water and agriculture managers }\end{array}$ \\
\hline U1-2 & $\begin{array}{l}\text { Source: processes of interest } \\
\text { Uncertainty: what are the minimum key } \\
\text { 'issues of concern' that should be included } \\
\text { in this model? } \\
\text { Nature: limited knowledge } \\
\text { Level: certainty, scenario }\end{array}$ & $\begin{array}{l}\text { Prioritize: central to model structure, so seek more information } \\
\text { Reduce: by stakeholder engagement on the topic of agricultural groundwater use } \\
\text { Describe: initial identification of all likely issues } \\
\text { Propagate: not yet defined } \\
\text { Communicate: acknowledge that issues will have to be prioritized } \\
\text { Anticipate: } \text { plan stakeholder engagement with water and agriculture managers }\end{array}$ \\
\hline U1-3 & $\begin{array}{l}\text { Source: definition of future conditions } \\
\text { Uncertainty: Which development activities } \\
\text { might drive changes in future groundwater } \\
\text { availability? } \\
\text { Nature: limited knowledge } \\
\text { Level: } \text { scenario }\end{array}$ & $\begin{array}{l}\text { Prioritize: significant risk, seek more information } \\
\text { Reduce: by engagement on the topic of agricultural groundwater use } \\
\text { Describe: identify potential future development activities for inclusion in model } \\
\text { Propagate: not yet defined } \\
\text { Communicate: acknowledge ambiguity about future development } \\
\text { Anticipate: plan stakeholder engagement with water and agriculture managers }\end{array}$ \\
\hline
\end{tabular}


conceptual model. All three uncertainties are at a 'scenario' level given that they present multiple options that cannot currently be described using bounds. Additional data could reduce the level of 'limited knowledge' uncertainty, but not necessarily for 'contradiction', which instead may be reduced by reaching a consensus (Guillaume et al., 2012). Given the central importance of uncertainties U1-2 and U1-3 in parsimonious qualitative structuring of a conceptual model, these were prioritized for reduction by collecting data through stakeholder engagement as detailed in Section 2.3. It was also hoped that by engaging stakeholders on the topic of U1-1, more detail on underlying assumptions might help in proposing a consensus on the relationship between groundwater and livelihoods. At this qualitative stage, it is not yet decided how these uncertainties will be propagated and represented in the communication of model results.

\subsection{Stakeholder engagement and cognitive mapping}

Analysis of stakeholder interviews by cognitive mapping generated two combined maps (simplified for demonstration purposes in Figure 2) linking agricultural groundwater use and the goals expressed by the interviewees: (12) farmers profit from agriculture, and (10) farmers demand smaller volumes of water. Domain analysis of cognitive map structure reveals the concepts most central (the largest number of linkages) to a perspective, and so could be used as a rationale to reduce the level of uncertainty U1-2 from 'scenario' (multiple sets of appropriate minimum concepts identified across perspectives) to 'certainty' (an agreed, single set of concepts that must be included in a meaningful conceptual model). For this case study, the single set of domaincentric concepts were: (6) farmers pump water when and where they need it, (8) farmers diversify crop choices, (15) groundwater level drops and (7) farmers pay low price to pump.

In response to uncertainty U1-1, comparisons of assumptions between both maps showed similar perceptions about what system states led to 'risk' versus 'opportunity'. The 'risk' was perceived to arise when (8) farmers grow only rice, leading to (10) farmers demand large volumes of water, with an assumption that this leads to a reduction of (11) adequate water volumes available in dry season. The 'opportunity' was perceived to arise when (9) surface water infrastructure is provided or when (8) farmers diversify crop choices (assuming this leads to (10) farmers demand small volumes of [ground] water). The sustainability of groundwater use was perceived to be an indirect outcome of farmers cropping choices that themselves were perceived to be affected by a variety of drivers. The ambiguity/'contradiction' uncertainty therefore arose from different perceptions about what drivers were controlling future agricultural development and whether these were weighted towards opportunity or risk, actually aligned with U1-3. Some stakeholders believe the dominant driver of agricultural groundwater use would be market value chain-based interventions by donors (Figure 2a) (e.g. (5) $N G O$ supports market) while others perceived the dominant driver to be direct, regulatory action by the government (Figure 2b) (e.g. (17) government implements Water Law licensing). This improved detail indicated that U1-3 was the primary uncertainty of concern and could be reframed as a 'contradiction' based on the perspectives identified by the cognitive mapping. The agreements on system structure allowed U1-1 to be ignored, and a
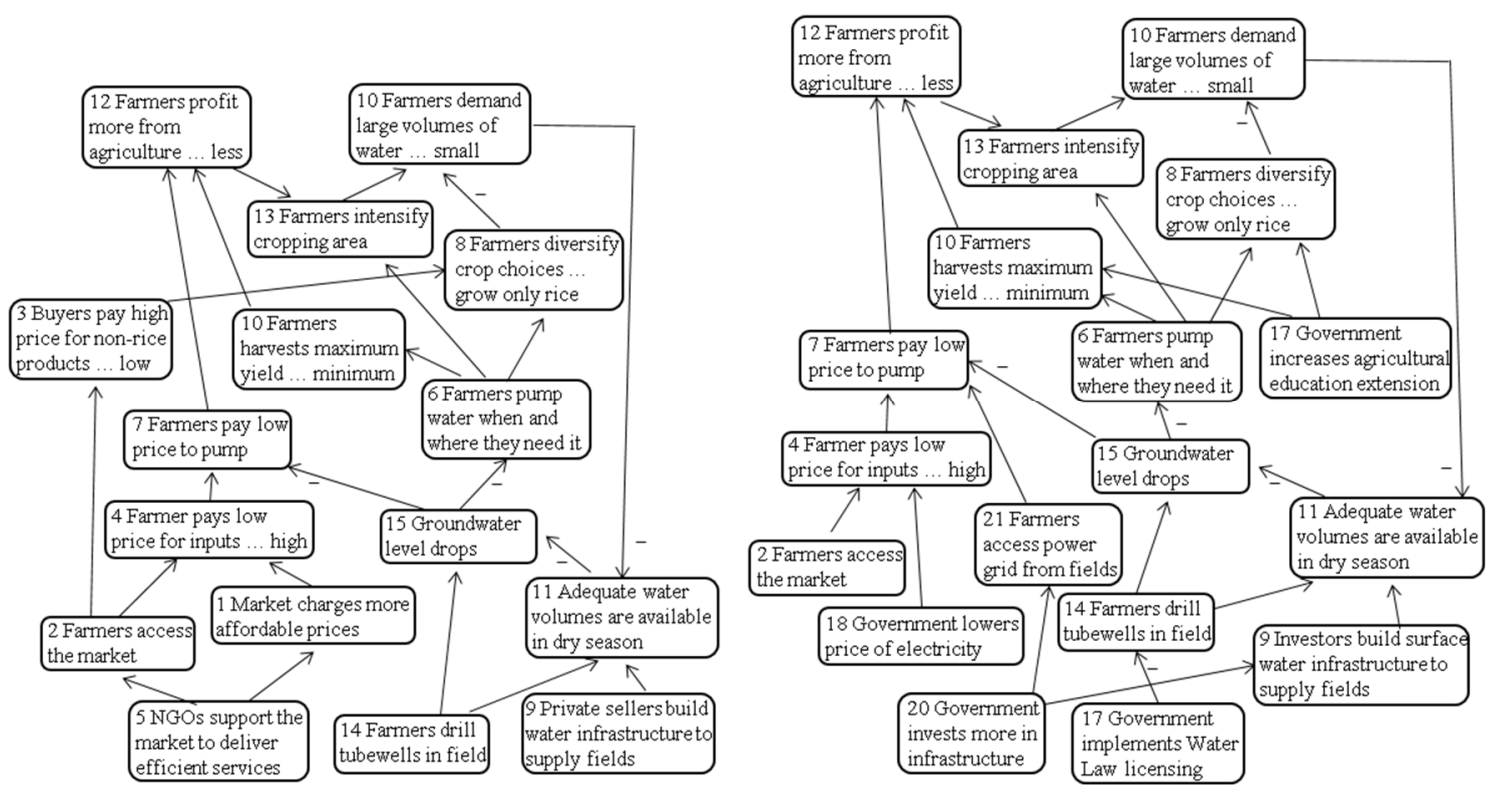

Figure 2. Two perspectives on groundwater use differentiated by the dominant development and resource management approach that they assume: a) market-based and b) policy-based. 
new uncertainty to be identified around validating the perceived causal linkages among system components (U2-1). An example is questioning the stakeholder assumption that crop diversification will lower irrigation demand. This could be tested at the quantification stage of model development to identify any tipping points, which could help with parsimonious model design.

The combined maps also identified multiple or conflicting causal pathways among concepts, indicating the perceived complexity of system structure. An example from the case study is demonstrated in Figure 2 a by the dual linkages between (5) NGOs support the market to deliver efficient services and (4) Farmer pays low price for inputs. Perceived causal pathways were firstly the idea that a supported market would lead to more (2) farmers access the market and/or secondly that (1) market charges more affordable prices because of increased efficiency. Mapping these multiple pathways means they can be transparently acknowledged, communicated and the importance of making them explicit within the final conceptual model can be negotiated.

\subsection{Second iteration of uncertainty framework: problem framing}

Reflecting on Iteration 1, the stakeholder engagement and cognitive mapping process has reduced the level of uncertainty around U1-1 by adding more data about the incidence of issues across stakeholder cognitive maps. Adding detail has allowed U1-2 and U1-3 to be reframed as multiple 'contradiction' uncertainties to be managed as the model process shifts to a 'problem framing' phase and boundaries are chosen for the conceptual model. Following from Section 3.2, a selection of these uncertainties is shown in Table 2. Managing these uncertainties requires making judgements on whether to accept a linkage assumption as correct or prioritize it for further validation (e.g. U2-1), whether a given concept should be included or excluded for model parsimony (e.g. U2-2), and whether to encourage a choice between contested future drivers or build scenarios with both (U2-3). Some may be handled qualitatively (build U2-3 drivers into conceptual model structure), some may be quantitatively validated by further research (U2-1) while others might be 'parked' and re-visited once the conceptual model is quantified (e.g. U2-2).

Table 2. Uncertainty management framework applied to problem framing phase

\begin{tabular}{|c|c|c|}
\hline & Uncertainty typology & Incertainty management tasks \\
\hline U2-1 & $\begin{array}{l}\text { Source: representation of system behavior } \\
\text { (continued from U1-1) } \\
\text { Uncertainty: will diversifying crops actually } \\
\text { result in a 'small' irrigation demand? } \\
\text { Nature: limited knowledge } \\
\text { Level: bounds }\end{array}$ & $\begin{array}{l}\text { Prioritize: seek more information } \\
\text { Reduce: by building or sourcing a crop water model, check bounds on arable land } \\
\text { Describe: model the extreme bounds of diversified crop water demand across region } \\
\text { Propagate: test the extreme bounds as model inputs } \\
\text { Communicate: report results as bounds } \\
\text { Anticipate: plan to source/adapt an existing crop model }\end{array}$ \\
\hline $\mathrm{U} 2-2$ & $\begin{array}{l}\text { Source: representation of stakeholder values } \\
\text { (continued from U1-2) } \\
\text { Uncertainty: Are efforts to reduce prices for } \\
\text { farmers dominated by improved market } \\
\text { access or supplier efficiency approaches? } \\
\text { Nature: limited knowledge } \\
\text { Level: bounds }\end{array}$ & $\begin{array}{l}\text { Prioritize: do not seek more information } \\
\text { Reduce: by sourcing likely bounds of effectiveness from literature and/or expert } \\
\text { elicitation, delay and revisit quantitatively } \\
\text { Describe: test sensitivity of 'farmer pays low price' to the extremes of each driver } \\
\text { Propagate: not yet defined } \\
\text { Communicate: report the sensitivity } \\
\text { Anticipate: decide if either driver is to be excluded based on testing }\end{array}$ \\
\hline U2-3 & $\begin{array}{l}\text { Source: representation of future conditions } \\
\text { (continued from U1-3) } \\
\text { Uncertainty: will market or policy dominate } \\
\text { Cambodia's agricultural development? } \\
\text { Nature: contradiction } \\
\text { Level: scenario }\end{array}$ & $\begin{array}{l}\text { Prioritize: do not seek more information } \\
\text { Reduce: (contradiction is inherently not reducible) } \\
\text { Describe: implement both of the possible approaches } \\
\text { Propagate: include both development drivers as model scenarios } \\
\text { Communicate: report on scenarios separately } \\
\text { Anticipate: } \text { suggest revision of plan if one approach becomes apparent over time }\end{array}$ \\
\hline
\end{tabular}

\section{DISCUSSION AND CONCLUSIONS}

Application of the UMF to the scoping and problem framing phases of the case study encouraged questioning of what was known and not known, and what boundaries and internal causal structures should be made explicit in a conceptual model to explore different water use tradeoffs. The UMF approach to conceptual modelling: encouraged clarity on what options were available to handle ambiguity and how it might affect problem framing and provided transparency through an iterative, structured process that communicated uncertainties about the modelling choices to be made. Deliberation, transparency, and awareness of resource limits all generated confidence that the resulting system abstraction was defensible and 'enough' to progress through the modelbuilding process. The prioritize task kept resource constraints front-of-mind so that stakeholder engagement could be targeted to those uncertainties most likely to threaten continued trust in the modelling process. The addition of data through the cognitive mapping analysis then clarified differences of perception about causal linkages within the system and the dominant drivers, while confirming that in fact there was overall agreement 
about the system structure. This provided a basis for pursuing parsimony which could be acted upon from the second iteration of the UMF. Given resource constraints, it was found to be easier to compare and prioritize actions in pursuit of the single overarching goal of uncertainty management compared to a multitude of best practice recommendations which do not explicitly guide action. The flexibility of the UMF allowed for multiple methods to be considered as suited the context and resources.

Some consideration was necessary around how to apply the UMF, such as how to trigger the identification of uncertainties, by what criteria to prioritize uncertainties for reduction, and finding methods appropriate for each type of uncertainty. There was concern about how best to approach these considerations, but this is not unique since these types of decisions must be made during the design of the modelling process regardless of whether an uncertainty management approach is taken or not. The documentation suggested by the UMF was somewhat onerous but aligns with best practice recommendations by Robinson (2015) to ensure future users of the model and stakeholders can track the decisions taken and why.

The focal point of uncertainty may provide a common goal to unite modellers and non-modellers in pursuit of best-practice conceptual models. An initial challenge to this can be communicating the concept of uncertainty. Narrative-building and a shift to more engaging and relatable language around uncertainty management is recommended for use with non-modeller stakeholders to facilitate their adoption of the concept. Discussions around uncertainty early in the modelling process may also help in setting realistic stakeholder expectations of what can be achieved, which is important for relationship-building, trust and transparency. Other uncertainty management approaches may be harnessed to similarly guide conceptual modelling, including Guillaume's Iterative Closed Question Modelling methodology and the work of Brugnach et al. (2008) and Refsgaard et al. (2007). It is hoped that the utility and holistic nature of this approach will encourage a stronger and more explicit focus on uncertainty during the scoping, problem framing and conceptualisation phases of IAM.

\section{ACKNOWLEDGEMENTS}

Thank you to my supervisors Tony Jakeman and Wendy Merritt for their guidance, to Sondoss El Sawah for support with cognitive mapping and to the Australian Government Research Training Program Scholarship.

\section{REFERENCES}

Argent, R.M., Sojda, R.S., Guipponi, C., McIntosh, B., Voinov, A.A., Maier, H.R. (2016). Best practices for conceptual modelling in environmental planning and management. Environmental Modelling \& Software 80, 113-121.

Brugnach, M., Pahl-Wostl, C., Lindenschmidt, K.E., Janssen, J.A.E.B., Filatova, T., Mouton, A., Holtz, G., van def Keur, P., Gaber, N.N. (2008). Complexity and Uncertainty: Rethinking the Modelling Activity, in: Environmental Modelling, Software and Decision Support: State of the Art and New Perspectives, Elsevier, Amsterdam.

Eden, C. (1994). Cognitive mapping and problem structuring for system dynamics model building. System Dynamics Review, 10 (2-3), 257-276

Erban, L.E., Gorelick, S.M. (2016). Closing the irrigation deficit in Cambodia: implications for transboundary impacts on groundwater and Mekong River flow. Journal of Hydrology, 535, 85-92

Guillaume, J.H.A. (2014). Managing uncertainty in modelling of wicked problems: theory and application to Sustainable Aquifer Yield. PhD Thesis, Australian National University, Canberra.

Guillaume, J.H.A., Qureshi, M.E., Jakeman, A.J. (2012). A structured analysis of uncertainty surrounding modeled impacts of groundwater-extraction rules. Hydrogeology Journal, 20, 915-932.

Hamilton, S.H., ElSawah, S., Guillaume, J.H.A., Jakeman, A.J., Pierce, S.A. (2015). Integrated assessment and modelling: Overview and synthesis of salient dimensions. Environmental Modelling \& Software, 64, 215229.

Jakeman, A.J., Letcher, R.A., Norton, J.P. (2006). Ten iterative steps in development and evaluation of environmental models. Environmental Modelling \& Software, 21, 602-614.

Refsgaard, J.C., van der Sluijs, J.P., Højberg, A.L., Vanrolleghem, P.A. (2007). Uncertainty in the environmental modelling process - A framework and guidance. Environmental Modelling \& Software, 22, 1543-1556.

Robinson, S. (2015). A tutorial on conceptual modelling for simulation, in: Proceedings of the 2015 Winter Simulation Conference. IEEE Press, pp. 1820-1834.

Walker, W.E., Harremoës, P., Rotmans, J., van der Sluijs, J.P., van Asselt, M.B., Janssen, P., Krayer von Krauss, M.P. (2003). Defining uncertainty: a conceptual basis for uncertainty management in model-based decision support. Integrated assessment, 4, 5-17. 